\title{
Brain-CAVE Interface Based on Steady-State Visual Evoked Potential
}

\author{
Hideaki Touyama \\ The University of Tokyo \\ Japan
}

\section{Introduction}

Recently, a new modality of the human-computer interface has been more and more emerging; The Brain-Computer Interface (BCI). The BCI is a communication channel, which enables us to send commands to external devices by using human brain activities (Wolpaw et al., 2002). As one of the remarkable achievements, we can see the report on invasive Brain-Machine Interface (BMI) (Hochberg et al., 2006).

Besides the technique of the invasive BMI, there are several types of non-invasive approaches for the brain signal acquisitions; for example, functional magnetic resonance imaging (fMRI), near infrared spectroscopy (NIRS), etc. Non-invasive methods have been noteworthy owing to the recent development of the signal processing method as well as acquisition apparatus. The performance of the BCI system is going to be improved and the applications has been seen; for example, the communication tool for people with disability, virtual reality games, and so on. Among them, the electroencephalography (EEG) has been investigated as one of the candidates for the low-cost and portable BCI system. It is well known that the EEG activities can be detected by the scalp recording, which are typically the order of 5-10 micro volts of potentials. The BCI system can extract the specific temporal and spatial patterns from the brain potentials, and translates them into the commands to control the machine according to the users' intent.

A variety of brain activities has been reported so far in the context of the BCI systems based on EEG signals; for instance, motor related potential (Pfurtscheller \& Neuper, 1997), event related P300 evoked potential (Farwell \& Donchin, 1988), visual evoked potential (VEP) (Kuroiwa \& Celesia, 1981), etc. With such brain activities, many applications have been developed in laboratories such as a virtual keyboard or joystick. However, most of them were studied on the system with the simple visual feedback involving a normal computer monitor.

The purpose of this book chapter is to show the technique to realize a BCI system in virtual reality environment and to suggest the possibility of the online control of computergenerated objects. Note that the most advantage of testing the BCI system in virtual reality is that we can easily test and simulate procedures for the $\mathrm{BCI}$ applications in reality (Pfurtscheller et al., 2006). Our works are based on the VEP, which is expected to yield high performance BCI systems. In spite of the expected use of the VEP, the BCI system based on such EEG oscillations has never reported in immersive virtual environments. 
In the next section, we briefly review the virtual reality technology including the immersive projection technology. In section 3, the previous works on a variety of $\mathrm{BCI}$ systems are presented. In section 4, we explain the visual stimuli in our experiments and report the performance in inferring the users' eye-gaze directions from the brain signals. We state about the results of the online controls of a stereoscopic virtual panorama. Finally, discussions and future works are followed.

\section{Virtual Reality}

Virtual reality is a technology with which the user can interact with a computer-generated environment; The virtual environment. There are seven concepts which are required for the virtual reality: simulation, interaction, artificiality, immersion, telepresence, full-body immersion, and network communication (Heim, 1993). In the context of the virtual reality, a variety of special devices have been newly developed; the data glove or data suit etc. The essence of these concepts may be familiar even with people who don't work with the technology.

The virtual environments are mainly provided by the visual stimuli displayed on a computer monitor, a head mounted display, or other special devices realizing stereoscopic images. However, the modality is not restricted to the visual one, owing to the recent understanding of the human perception and the development of the special 'displays'. There are several types of sensory information to obtain such virtual experiences; auditory, haptic, olfactory, and other possible sensations. For example, the force sensation is well experienced by a force display.

There are a lot of applications using the virtual reality technology: For example, the modelling and visualization of the invisible phenomena and the experiences of them in the simulations, the surgical applications sometimes involving telepresence or telexistance, the remote operations of the industrial machine, prototyping or mock up of the developing products, the educational use, the entertainments such as games, the applications for mental therapy, and so on.

There are remarkable advantages in the use of the virtual reality technology. The technology can easily provide the user with the safety and reproducibility owing to the artificially simulated environment. Furthermore, we can reduce the cost and other possible resources.

Among the technologies of the virtual reality, there is a system which provides the users with high degree of immersion: The CAVE (Computer Augmented Virtual Environment). The original CAVE was developed by the group of the University of Illinois and demonstrated at the SIGGRAPH (Cruz-Neira et al., 1993). This type of display system has been designed to perform the activities in a variety of use as mentioned above. The descendant systems have been developed all over the world based on the novel concept and technology of the CAVE. The fundamental technologies of CAVE-like display system will be briefly explained later.

The users can interact with virtual objects by using the standard input devices like a joystick, game controller or newly developed devices such as a data glove. The interaction can be in real time to reflect the input information appropriately to the system. It is sometimes realized by sensing the physical states of the user; for example, using the motion capturing system with which the position or movement of the users' head or entire body can be detected.

Recently, the BCI system has been studied with virtual reality (Bayliss, 2003; Bayliss \& Ballard, 2000; Friedman et al., 2004; Friedman et al., 2007; Lalor et al., 2003; Leeb et al., 2005; Ron-Angevin et al., 2005; Scherer et al., 2007; Fujisawa et al., 2008a), which enables people to interact with the virtual environment using the human brain activities: with no standard 
input devices. For example, by using a head mounted display, Bayliss et al. investigated P300 evoked potential (Bayliss, 2003; Bayliss \& Ballard, 2000). The group of Graz University of Technology and the UCL has been studying the 'walking from thought' in CAVE-like system using motor imagery tasks (Friedman et al., 2004; Friedman et al., 2007; Leeb et al., 2005). Most advantage of testing the BCI system in virtual reality environments is that we can easily test and simulate procedures for the BCI applications in reality (Pfurtscheller et al., 2006). However, except for the BCI system based on motor imagery tasks, such applications have never been implemented into immersive virtual reality environment. This book chapter will focus on the Brain-CAVE Interface based on the VEP.

\section{Previous Works on $\mathrm{BCl}$}

There are two types of signal aquisition in the context of the interface based on the brain activities; The invasive and non-invasive. The invasive BCI, which is often called as BMI, has been developed for people with disability. Neurosurgery enabled a person to control an artificial hand using the Cyberkinetics Neurotechnology's BrainGate as well as the operation of a computer cursor, a variety of swithing operations of lights etc (Cyberkinetics, Inc.). In the BMI system, the electrodes are directly implanted into the brain. Therefore, high quality of the brain signals can be obtained.

The most advantage of the non-invasive approaches is that we can be blessed with the system in our ordinary life. For able-bodied people, the demand on the non-invasive BCI system will be more and more enlarged. In fact, the low-cost products of the non-invasive $\mathrm{BCI}$ systems have been developed today. Of course, there is no risk of neurosurgery in such systems and thus it is comparably easy to prepare for the use, while the non-invasive approaches produce poor signal resolution.

There are several types of non-invasive BCI systems; for example, functional magnetic resonance imaging (fMRI) (Buxton, 2002; Huettel et al., 2004), near infrared spectroscopy (NIRS) (Watanabe et al., 1996), etc. Remarkable achievements have been reported on these approaches in laboratories. Based on the decoding technology (Kamitani \& Tong, 2005) in the fMRI study, the system could infer the shape of the users' hand (among three states of scissors, paper, and rock) with $85 \%$ of the correct rate and a seven second delay, and then could control the robot hand in online (Tech-on, 2006). The NIRS study showed the possibility to control a model train by mental arithmetic task resulting in the haemodynamic response; The optical-BCI system (Utsugi et al., 2007). However, in the present stage these measurement apparatus give high cost and no portability.

The Electroencephalography (EEG) was reported by Hans Berger in 1929. Since then, it has been the most studied method among the non-invasive measurements. The advantage of the EEG in the context of the BCI is; its high temporal resolution, ease for practical use, low-cost, and portability. The low spatial resolution may be the disadvantage. And the artefact or environmental noise tend to reduce the performance of the BCI system. However, the BCI system based on EEG is prosperous owing to the recent extensive studies on the signal processing which may cover the disadvantage.

The P300 response is the event related potential which can measure the degree of concentration of the subject on the specific stimulus. Farwell et al. investigated a P300 speller, which enabled the user to type strings only by brain activities of P300 responses (Farwell \& Donchin, 1988; Krusienski et al., 2008). The $6 \times 6$ matrix of letters flashing randomly were presented on the computer display. The user selected ' $\mathrm{A}$ ' by counting the 
number of times that the letter 'A' flashed. A variety of applications has been studied using this type of evoked potentials. Note that Bayliss showed the P300 responses could control the virtual objects (Bayliss, 2003; Bayliss \& Ballard, 2000) .

The mu rhythm of somatosensory cortices was found owing to the recent development of computer-based analyses on EEG activities. Movement and even phantom movement are accompanied by a suppression of mu and beta rhythms. This suppression has been known as event-related de-synchronization (ERD). After the movements or when inactive, the idling rhythm increase call as event-related synchronization (ERS) occurs, as in the case of visual alpha rhythm during eye close in relax. It is a strong motivation for EEG-based brain computer interfacing (Pfurtscheller \& Neuper, 1997).

A lot of laboratory has developed the BCI system based on the ERD/ERS modulation (Wolpaw \& McFarland, 2004; Blankertz et al., 2006; Pfurtscheller et al., 2006). The group of Wolpaw demonstrated the operation of one and two dimensional cursor on a computer screen (The Wadsworth BCI) (Wolpaw \& McFarland, 2004). However, prior to the experiments, the participants had to learn to control their own mu and beta rhythms. The BCI system of the group of Graz University of Technology and UCL (Pfurtscheller et al., 2006) is also based on the motor imagery. The BCI system has been implemented in immersing virtual environment (ReaCTor, which is a CAVE-like system). The walking from thought was demonstrated. The subjects participated with many runs of the BCI control in a variety of experimental environments using PC, head-mounted display, and CAVE. Note that the novel works on motor imagery and the $\mathrm{BCI}$ system based on it are reviewed in the report of (Wolpaw et al., 2002).

Most of the previous studies on BCI systems has been pefromed in the ideal environments. In the present stage the performance of the BCI system has been more and more improved. However, it is important to study the systems in the simulated environments in order to extract the problems in future practical use in reality. Our concern is in the simulated environment to examine the performances of the BCI system.

\section{The BCI Based on SSVEP}

\subsection{Visual Evoked Potential}

Let us see the mechanism of the BCI system based on the VEP. The VEP is an event driven response to an external visual stimulus that is observed on visual cortex (Kuroiwa \& Celesia, 1981). In general, if a subject undergoes a flickering visual stimulus with the flickering frequency more than 4 or $5 \mathrm{~Hz}$, the steady-state responses can be obtained; The steady-state VEP (SSVEP), which is the synchronized signals with the flickering frequency often accompanied by the harmonic ones. This type of EEG oscillations has been proven as a reliable signal for the control of a BCI system.

Vidal introduced a BCI system based on the VEP (Vidal, 1973). The system could infer the users' eye-gaze directions in order to determine the direction in which the users wished to move a cursor. Furthermore, Middendorf et al. (Middendorf et al., 2000), Cheng et al. (Cheng et al., 2002), and Trejo et al. (Trejo et al., 2006) also reported a feasibility of such systems to determine the eye-gaze directions. In these works, several checkerboard patterns or virtual buttons (more than 10 buttons in the report of (Cheng et al., 2002)) appear on a computer monitor or a LED-based display and flash at different rates. When the user gazes at an interested flickering button, the system determines the frequency of the steady-state response. 
The performance of the BCI system depends both on the speed and the accuracy. In general, the VEP yields high information transfer rate (Wolpaw et al., 2002). In fact, the group of Tsinghua University reported the advantage of EEG oscillations of SSVEP (Hong, 2007). The user could input the sequence of interested phone numbers on the virtual telephone using LED-based visual stimuli. The information transfer rate reached to 55 bits/min (Cheng et al., 2002).

\subsection{Immersive Virtual Environment}

The design of the visual stimuli is one of the key for the reliable BCI system based on the VEP. In this study, we adopted the visual stimuli in immersive virtual environment.

One of the features of CAVE display system (Cruz-Neira et al., 1993) is its multi-screen configuration. The viewing angle of the user is remarkably enlarged, compared to normal computer monitors. The image displayed in CAVE is interactive. For instance, the user can change the views of the images in real time according to the users' viewpoint measured by the position tracker and also via input devices such as game controllers. These input data are transmitted to the graphics workstations from the input devices. Since the LCD shutter glasses are used to generate stereoscopic images, shutter timing of the glasses must be synchronized with the scanning of the screens.

We have experimented with our BCI system in an immersive virtual environment in the University of Tokyo. Figure 1 shows the external appearance of the projection system that is a cubic shape with five screens positioned at the front, the left, the right, the ceiling, and the floor. The area of each screen is $2.5 \mathrm{~m} \times 2.5 \mathrm{~m}$. By using this type of projection system, the user can feel high degree of immersion. Therefore, the immersive virtual environment is expected to be a reasonable method to evaluate a BCI system in advance before the implementation into the real world, extracting a variety of problems in practical use. Nevertheless, there has been no extensive study on the Brain-CAVE Interface but only the works on motor related potentials.

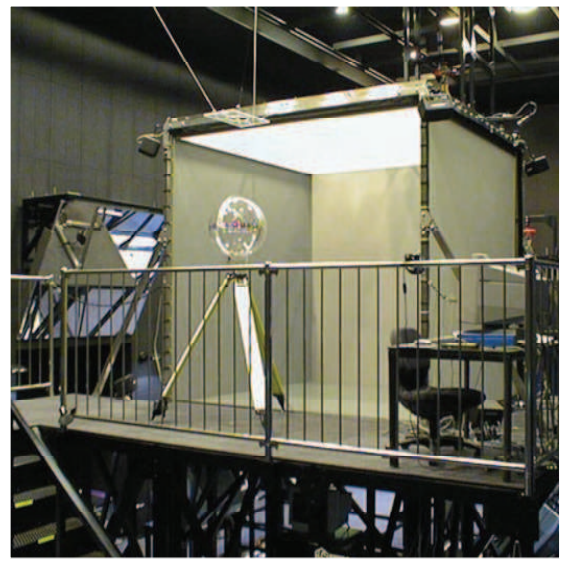

Figure 1. The external appearance of a projection system to generate an immersive virtual environment. The system has a viewpoint tracking apparatus and LCD shutter glasses for appropriate stereoscopic images to make the users feel high degree of immersion

Two flickering virtual buttons were prepared being superposed on a $3 \mathrm{D}$ virtual panorama in immersive virtual environment. The configuration is illustrated in Figure 2. Each button had 
the visual angle more than 10 degrees of both in horizontal and vertical at a view distance of $2 \mathrm{~m}$. The subjects were instructed to gaze at a fixation point on either buttons. The flickering frequencies were selected between 4 and $8 \mathrm{~Hz}$ so as to synchronize with the refresh rate of vertical scans of the CAVE-projector. Note that this range of flickering frequency had been found to yield clear SSVEP, while the frequency more than $20 \mathrm{~Hz}$ resulted in unclear brain responses. The viewpoint tracking system was activated during experiments.

Note that in previous studies on the SSVEP, the visual angle for the visual stimulus was at most a few degrees, being restricted on the size of the usual computer displays. On the other hand, the CAVE system would have an advantage of large visual angles and a variety of visual stimuli would be arranged in the virtual space.

When the user gazed at one of the two flickering objects, the other stimulus was still in the visual field. This is an interesting problem related with the selective attention for the specific visual stimulus, which will be mentioned later.

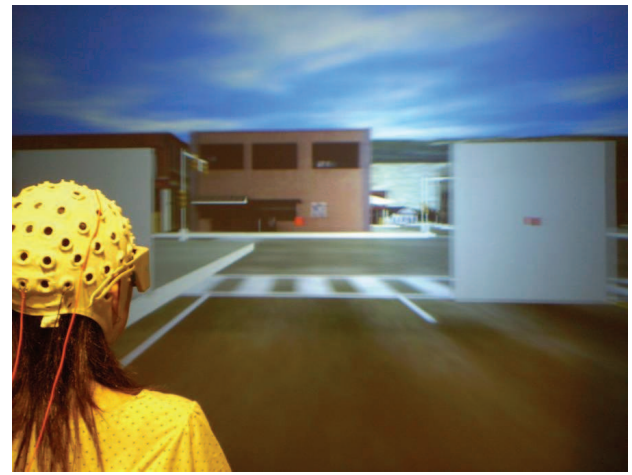

Figure 2. The left and right flickering (white/black) visual stimuli with square shape superposed on a front screen and a subject sitting in immersive virtual environment. These two flickering frequencies have different rates

\subsection{EEG Recordings}

The healthy volunteers (s1-s4) with normal or corrected to normal vision participated in the experiments as subjects (range 22-36 years old). They were not trained for the EEG measurements with the flickering visual stimuli. During the experiments, each subject relaxed on an arm-chair facing the front screen of the immersive virtual environment, wearing LCD shutter glasses.

A modular EEG cap system was applied for scalp EEG recordings. Three-channel EEG signals were recorded from parieto-occipital and occipital; that is, PO7, PO8 and Oz according to the extended international 10/20 system (Jasper, 1958) as shown in Figure 3. A body-earth and a reference electrode were on a forehead and on a left ear lobe, respectively. The analogue EEG signals were amplified at a multi-channel bio-signal amplifier (MEG6116, NIHON KOHDEN, Inc. Japan). A notch filter was applied to reduce the $50 \mathrm{~Hz}$ power line interference. The amplified signals were band-pass filtered between 1.5 and $30 \mathrm{~Hz}$, and sampled at $100 \mathrm{~Hz}$ by using an A/D converter with a resolution of 16 bits. The digitized EEG data was stored in a standard personal computer.

There were two types of offline experimental tasks (task 1 and 2) were imposed to collect data sets for the later EEG-classification. The users were asked to gaze at and pay attention 
to a left visual stimulus in the task 1 and a right one in the task 2 . For all subjects, one experiment consisted of 5-10 sessions for each task. Each session lasted for 30 seconds. The session of the task 1 and 2 were performed by turns. After one session, one-minute rest was imposed. For several subjects, the experiments were repeatedly performed several times over several days. During these offline measurements the virtual panorama was not controlled (with no visual feedback).

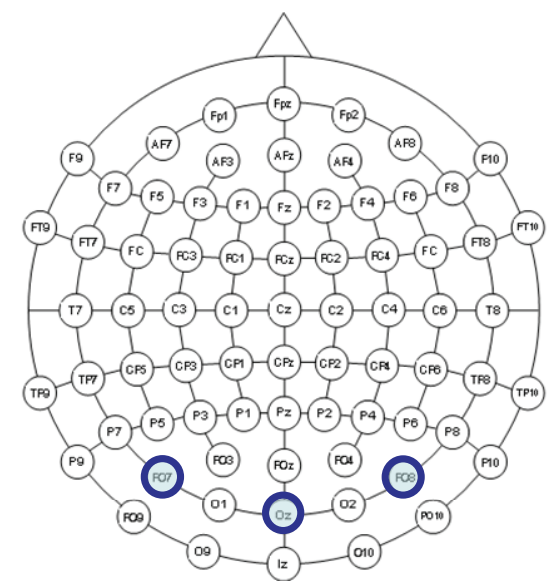

Figure 3. Location of electrodes to collect the SSVEP. Three-channel EEG signals were recorded from parieto-occipital sites (PO7 and PO8) and occipital (Oz) according to the extended international 10/20 system

\subsection{Classification of EEG Oscillations}

After the data acquisitions, the recorded EEG signals were at first analyzed in offline. Frequency analysis was applied to extract the expected EEG oscillations. The analyzing time period and the window function were fixed to 2 seconds and Hanning, respectively. The EEG features were extracted from the linear combination of voltage value between three electrodes $[\mathrm{V}(\mathrm{Oz})-\{\mathrm{V}(\mathrm{PO})+\mathrm{V}(\mathrm{PO})\} / 2]$, expecting the reduction of the environmental noise or possible artefacts, where $\mathrm{V}(\mathrm{E})$ means the voltage value detected at the scalp-electrode $\mathrm{E}$. The typical power spectral densities in average are shown in Figure 4. The harmonic signals of the SSVEP were observed. As for one subject s4, the SSVEP was not clear.

The SSVEP was observed at 16 and $18 \mathrm{~Hz}$, which was expected to be harmonic signals induced by 8 and $6 \mathrm{~Hz}$ of flickering frequency, respectively. Therefore, wide range of the power spectral densities including these frequencies (range 3-25 Hz) were considered in single trial (non-average) EEG data to evaluate the classification performance discussed below. The spontaneous EEG signals of alpha rhythms (typically 8-13 Hz) were not excluded in these analyses. Note that in our previous study the alpha band contributed to three-class classification aiming the online navigations in the virtual world (Touyama \& Hirose, 2007a).

The algorithm of support vector machines (SVM) (Vapnik, 2000) with linear kernel was applied to classify two states of brain activities (during gazing at left stimulus and right one, respectively). To estimate the performance, a leave-one-out method was applied, where only one data is used for testing and the others are for training. 


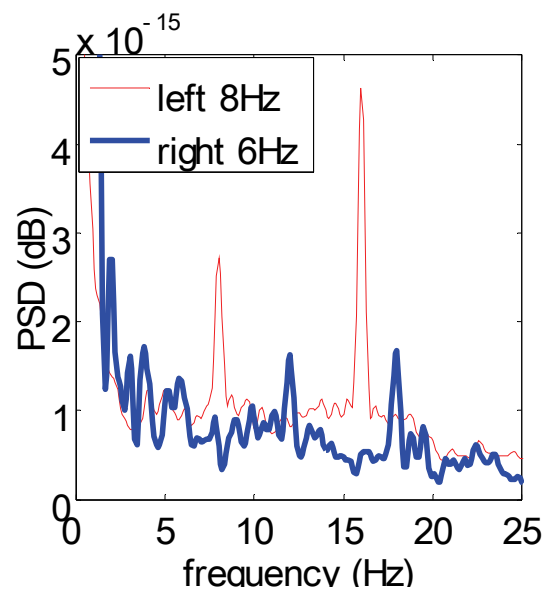

Figure 4. Typical result of the average power spectral densities (PSD). The flickering frequencies are 8 and $6 \mathrm{~Hz}$ for left and right visual stimulus, respectively

The results of the classification are shown in Table 1 . The performances were found to be 86.6, $71.4(80.0,78.0)$, and $81.0(82.0) \%$ for the subjects s1, s2, and s3, respectively. The grand average of three subjects was $79.9 \%$. The numbers in the parentheses denote the results of different experimental days.

Now the maximum information transfer rates can be estimated for three subjects with offline results above, while the rate is strongly dependent upon the application design. The rates are dependent on both speed and accuracy, and defined by the following equation (1) (Wolpaw et al., 2002). If a trial has $\mathrm{N}$ possible selections and each selection has the same probability of being the one that the user desires, if the probability $\mathrm{P}$ that the desired selection will actually be selected is always the same, and if each of the other selections has the same probability of being selected, the bit rate $B$ can be expressed as

$$
B=\log _{2} N+P \log _{2} P+(1-P) \log _{2}[(1-P) /(N-1)] .
$$

This equation yielded the maximum information transfer rate between 4.1 and $13.0 \mathrm{bits} / \mathrm{min}$ from our offline measurements, which amounts to the standard performances (5-25 $\mathrm{bits} / \mathrm{min}$ ) of BCI today.

\begin{tabular}{|c|c|c|}
\hline $\begin{array}{c}\text { Subjects' } \\
\text { Name (day) }\end{array}$ & $\begin{array}{c}\text { Flickering Frequency (Hz) } \\
\text { (left, right) }\end{array}$ & $\begin{array}{c}\text { Classification Performances } \\
(\%)\end{array}$ \\
\hline $\mathrm{s} 1(1)$ & $(8.0,6.0)$ & 86.6 \\
\hline $\mathrm{s} 2(1)$ & $(8.0,6.0)$ & 71.4 \\
\hline $\mathrm{s} 2(2)$ & $(8.0,6.0)$ & 80.0 \\
\hline $\mathrm{s} 2(3)$ & $(6.9,4.8)$ & 78.0 \\
\hline $\mathrm{s} 3(1)$ & $(8.0,6.0)$ & 81.0 \\
\hline $\mathrm{s} 3(2)$ & $(6.9,4.8)$ & 82.0 \\
\hline Avr. & - & 79.9 \\
\hline
\end{tabular}

Table 1. Classification performances (percent corrects) for two conditions of flickering frequencies. The number with subjects' name denotes the experimental day. For example, s3(2) denotes the subject $\mathrm{s} 3$ on the $2^{\text {nd }}$ experimental day 


\subsection{Binary Controls}

We will show here the experimental results on the online control of a virtual panorama in the immersive virtual environment. After the data acquisition at an amplifier, the digitized EEG data was transmitted to a signal processing server through the network. At the server, the two-class classification mentioned before was performed by using latest 2 seconds of data. The results of the classification (binary control commands) from the brain activities on visual cortex ( 1 or $r$ corresponding to the left or right flickering stimulus, respectively) were transmitted to the workstation of the immersive virtual environment also through the network. At this workstation, both the images of panorama (a virtual city including roads, buildings, trees, sky, etc.) and the flickering stimuli for each eye were independently generated by using the library of OpenGL Performer (SILICON GRAPHICS, Inc.) to reflect on the screen. The user observed the visual feedback of stereoscopic images through the LCD shutter glasses (with visual feedback).

It is well known that there is a speed-accuracy trade off in the BCI system. There were two types of online experiments in this study to demonstrate the trade off. One is the online system with a consecutive counter (Cheng et al., 2002), and the other is without it. With this counter, the control command was set to $r$ only if the result of the classification was more frequently recognized than 1 for certain time period, and the same in the case of setting 1 command. In this study, the time period was set to 1 second.

With the flickering frequency of 6.9 (left) and $4.8 \mathrm{~Hz}$ (right), the subjects participated in the online experiments. The frequency combinations were same with our previous online studies. Before the experiments, the participants had no specific training and were only instructed to gaze at and focus attention on a red-coloured fixation point on the flickering stimulus. The eye-fixation point changed its position every 10 seconds between the left and right (as shown in Figure 2). One session lasted for 90 seconds. In the online experiments, the left and right visual stimuli play a key role in controlling a virtual panorama.

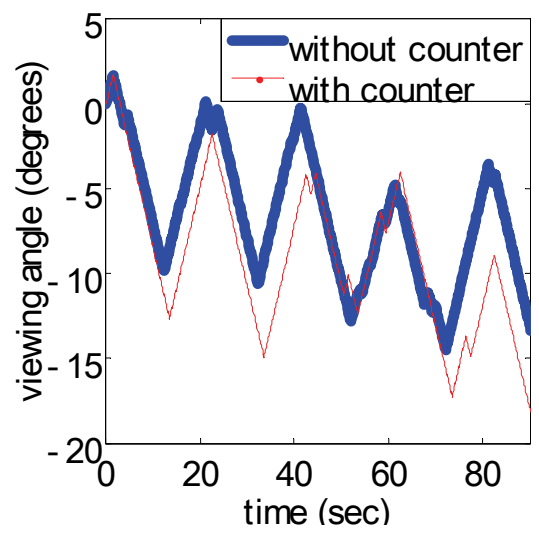

Figure 5. The typical result of the time dependence on the viewing angle of the subject in the virtual city. The flickering frequencies were 6.9 and $4.8 \mathrm{~Hz}$ for left and right flickering stimulus, respectively. The results with and without consecutive counter are illustrated

In Figure 5, the graphs show typical experimental results of the binary control of a virtual panorama. The plot shows the relation between the time and the viewing angle of the 
subject in the virtual city, which varies one after the other by the classification result from the signal processing server. Even without consecutive counter, the user could control the panorama well according to the subjects' intent. With the counter, the accuracy seems to be slightly improved, but the speed was modest, which demonstrated the trade off in the online system. In the online analyses, the average classification performance was $85 \%$.

\subsection{Discussions and Future Works}

This study presented a non-invasive BCI system based on the SSVEP in immersive virtual environment. The EEG oscillations, induced by two flickering virtual buttons superposed on the computer-generated panorama, were recorded and analyzed. The flickering frequencies were selected between 4 and $8 \mathrm{~Hz}$. Applying support vector machines, the single trial EEG data with 2 seconds of analyzing time yielded $85 \%$ of average classification performance in controlling the virtual panorama inferring the eye-gaze directions. The online demonstrations in immersive virtual environment showed a possibility to control the virtual objects according to the brain signals.

The previous study on BCI based on EEG in CAVE-like system investigated the walking in virtual environment using motor related potentials. The work in the report of (Pfurtscheller et al., 2006) required eight seconds of hand/foot imagery tasks to achieve two-class classification (estimated $1.5 \mathrm{bits} / \mathrm{min}$ of average information transfer rate), while non-cue based BCI system has been discussed elsewhere (Pfurtsheller et al.). On the other hand, the future advantage of our SSVEP-based BCI is in shortening of analyzing time period and sliding of the window which requires no cue for the user. It was reported that the information transfer rate reached to 55 bits/min (Cheng et al., 2002). Furthermore, the SSVEP-based BCI system requires no training for users, which is one more advantage. However, in our experiments, clear SSVEP could not be observed for one subject, which will be investigated again.

In this study, the binary classification rate was about $80 \%$ and $85 \%$ in offline and online, respectively. In our previous study, the rate was about $92 \%$ even with 1 second of analyzing time and occipital recordings, involving two virtual buttons floating in the dark virtual space (Touyama \& Hirose, 2007b). Thus, the condition of the visual stimulus is thought to influence the performance to a large extent. This is an important point in developing the BCI system based on SSVEP using the flickering stimuli superposed on the real scene, because the conditions of the images is in general varied rapidly. Therefore, the conditions of the visual stimulus would be systematically investigated in our future works in order to have clear and robust brain responses. As well as the flickering frequency, spacing, and size are required to be considered.

The improvement of the classification algorithm will be in our research scopes. In the report of (Trejo et al., 2006), kernel partial least squares (KPLS) algorithm was investigated to have high recognition rate of $80-100 \%$ in multi-class classification with 1-5 seconds of latencies. With this algorithm, the moving map display based on flickering checkerboard patterns was successfully controlled in a computer monitor. In the analyses in our study, we just adopted simple FFT analyses combined with SVM for the binary classification. It would be required to use only the power spectral densities corresponding to the flickering frequencies and their harmonic signals instead of all the power spectral densities between 3 and $25 \mathrm{~Hz}$. Such kind of feature selection would help the SVM to increase the classification accuracy. 
It is necessary to show a useful online application using our BCI system with multi-class classification in immersive virtual environment. One of the examples is to realize the free navigation (walk-through or fly-through) by the SSVEP as well as a manipulation of virtual objects or operation of menu windows. In our preliminary studies, three-class classification has been studied in the context of the navigation. The results for a subject are shown in the Table 2. The experimental settings were similar to that in this study. There, the Fishers' linear discriminant analyses showed about $74 \%$ of an average classification performance in inferring three eye-gaze directions, that is, left, right visual stimuli, and a centre eye-fixation point (Touyama \& Hirose, 2007a). The ultimate goal in such multi-class applications is that by using tiny visual stimuli arranged in the virtual space with the flickering frequencies near or more than critical one to realize more natural interaction.

\begin{tabular}{|l|c|c|c|}
\hline & Classified into left & Classified into right & Classified into centre \\
\hline Task left & $68.7[71.4]$ & $4.0[7.6]$ & $27.3[21.0]$ \\
\hline Task right & $5.3[14.3]$ & $69.4[65.2]$ & $25.3[20.5]$ \\
\hline Task centre & $6.7[13.8]$ & $5.3[6.7]$ & $88.0[79.5]$ \\
\hline
\end{tabular}

Table 2. The performance (percent correct) of three-class classification of EEG activities during gazing at left, right, and centre fixation point. The visual stimuli were similar to those of this study (involving two flickering stimuli superposed on the virtual panorama). Note that there was no flickering stimulus at the centre fixation point. The number out of [ ] (in [ ]) denotes the result on the $1^{\text {st }}\left(2^{\text {nd }}\right)$ experimental day

During the EEG measurements, the subjects were sitting on the luxury sofa and were instructed not to move. This gives the subjects both physical and mental fatigue. Aiming to realize free postures during EEG acquisitions, we started to analyse the SSVEP measurements during standing in the immersive projection system. The results for a subject are shown in Table 3. It was found that the SSVEP were clearly obtained and the rather high classification performance was achieved in three-class classification.

\begin{tabular}{|l|c|c|c|}
\hline & Classified into left & Classified into right & Classified into centre \\
\hline Task left & $81.4[81.4]$ & $9.3[1.3]$ & $9.3[17.3]$ \\
\hline Task right & $5.3[1.3]$ & $64.0[86.7]$ & $30.7[12.0]$ \\
\hline Task centre & $5.3[5.3]$ & $16.0[2.7]$ & $78.7[92.0]$ \\
\hline
\end{tabular}

Table 3. An example of the performance of three-class classification of EEG activities during standing. The subject gazed at left, right, and centre fixation point. There were two flickering stimuli floating in the dark virtual space. Note that there was no flickering stimulus at the centre fixation point. The number out of [ ] (in [ ]) denotes the result on the $1^{\text {st }}\left(2^{\text {nd }}\right)$ experimental day

There is a noteworthy topic in the context of independent BCI systems. The report of (Kelly et al., 2005) presented the data suggesting that the SSVEP can be used as a measure of visual spatial attention. This type of the independent BCI (see (Walpow et al., 2002)), which requires no eye-movement, would be one of the challenges of the EEG-based interfacing 
systems. We are performing the experiment on the EEG measurements with visual-spatial attention (Fujisawa et al., 2008b).

The study of the BCI system in immersive display has not been performed extensively so far. We hope that the Brain-CAVE Interface would be one of the research paradigms in the field of virtual reality and contributes to the advances in human-computer interaction.

\section{Acknowledgment}

This work was partly supported by Mizuho Foundation for the Promotion of Sciences.

\section{References}

Bayliss, J.D. (2003). The use of the evoked potentials P3 component for control in a virtual apartment, IEEE Transaction on Neural Systems and Rehabilitation Engineering, 11(2).

Bayliss, J.D. \& Ballard, D.H. (2000). A virtual reality testbed for brain-computer interface research, IEEE Transactions on Rehabilitation Engineering, 8(2), pp. 188-190.

Blankertz, B.; Dornhege, G.; Krauledat, M.; Muller, K.R.; Kunzmann, V.; Losch, F. \& Curio, G. (2006). The Berlin Brain-Computer Interface: EEG-based communication without subject training, IEEE Trans Neural Syst. Rehabil. Eng, 14(2), Jun, pp. 147-152.

Buxton, R.B. (2002). An Introduction to Functional Magnetic Resonance Imaging: Principles and Techniques, Cambridge Univ. Press, ISBN 0-52158-113-3.

Cheng, M.; Gao, X.; Gao, S. \& Xu, D. (2002). Design and Implementation of a BrainComputer Interface With High Transfer Rates, IEEE Transactions on Biomedical Engineering, 49(10), pp. 1181-1186.

Cruz-Neira, C.; Sandin, D.J. \& DeFanti, T.A. (1993). Surround-screen projection-based virtual reality: The design and implementation of the CAVE, ACM SIGGRAPH'93 Proc, pp. 135-142.

Cyberkinetics, Inc.

http://www.cyberkineticsinc.com/content/medicalproducts/braingate.jsp

Farewell, L.A. \& Donchin, E. (1988). Taking off the top of your head: Toward a mental prothesis utilizing event-related brain potentials, Electroenceph Clin Neurophysiol, 70, pp. 510-523.

Friedman, D.A.; Slater, M.; Steed, A.; Leeb, R.; Pfurtscheller, G. \& Guger, C. (2004). Using a Brain-Computer Interface in Highly-Immersive Virtual Reality, IEEE Virtual Reality Workshop.

Friedman, D.A.; Leeb, R.; Guger, C.; Steed, A.; Pfurtscheller, G. \& Slater, M. (2007). Navigating virtual reality by thought : What is it like ? Presence: Teleoperators and Virtual Environments, 16(1), pp.100-110.

Fujisawa, J.; Touyama, H. \& Hirose, M. (2008). EEG-based navigation of immersive virtual environments using common spatial patters, Proc. of IEEE Virtual Reality Conference 2008, pp.251-252.

Fujisawa, J.; Touyama, H. \& Hirose, M. (2008). Extracting Alpha Band Modulation during Visual Spatial Attention without Flickering Stimuli using Common Spatial Pattern, Proceedings of $30^{\text {th }}$ Annual International Conference of the IEEE Engineering in Medicine and Biology Society (EMBC'08), pp.620-623.

Heim, M. (1993). The Metaphysics of Virtual Reality, Oxford University Press. 
Hochberg, L.R.; Serruya, M.D. ; Friehs, G.M. ; Mukand, J.A. ; Saleh, M.; Caplan, A.H.; Branner, A.; Chen, D.; Penn, R.D. \& Donoghue, J.P. (2006). Neuronal ensemble control of prosthetic devices by a human with tetraplegia. Nature 442, pp. 164-171.

Hong, B. (2007). BCIs using EEG oscillations: Towards practical applications, International Workshop on Brain-Computer Interface Technology 2007.

Huettel, S.A.; Song, A.W. \& McCarthy, G. (2004). Functional Magnetic Resonance Imaging, Sinauer Associates, ISBN 0-87893-288-7.

Jasper, H.H. (1958). The ten-twenty electrode system of the International Federation, Electroencephalogr Clin Neurophysiol, 10, pp. 371-375.

Kamitani, Y. \& Tong, F. (2005). Decoding the visual and subjective contents of the human brain. Nature Neuroscience, 8, 5, pp. 679-685.

Kelly, S.P.; Lalor, E.C.; Reilly, R.B. \& Foxe, J.J. (2005). Visual spatial attention tracking using high-density SSVEP data for independent brain-computer communication, IEEE Trans Neural Syst Rehabil Eng, 13(2), pp. 172-178.

Kuroiwa, Y. \& Celesia, G.G. (1981). Visual evoked potentials with hemifield pattern stimulation, Their use in the diagnosis of retrochiasmatic lesions, Arch. Neurol. 38, pp. 86-90.

Krusienski, D.J.; Sellers, E.W.; McFarland, D.J.; Vaughan, T.M. \& Wolpaw, J.R. (2008). Toward enhanced P300 speller performance. J Neurosci Methods. Jan 15; 167(1): 1521. Epub 2007 Aug 1.

Lalor, E.; Kelly, S.P.; Finucane, C.; Burke, R.; Smith, R.; Reilly, R. \& McDarby, G. (2003). Steady-state VEP-based Brain-Computer Interface control in an immersive 3-D gaming environment, Proceedings of the EURASIP, 2003.

Leeb, R.; Scherer, R.; Keinrath, C.; Guger, C. \& Pfurtscheller, G. (2005). Exploring Virtual Environments with an EEG-based BCI through Motor Imagery, Biomedzinishe Technik, Berlin, 52, pp. 86-91.

Middendorf, M.; McMillan, G.; Calhoun, G. \& Jones, K.S. (2000). Brain-Computer Interfaces Based on the Steady-State Visual-Evoked Response, IEEE Transactions on Rehabilitation Engineering, 8(2), pp. 211-214.

Pfurtscheller, G. et al. Human brain-computer interface. In: Vaadia,E., Riehle,A.(Eds.), Motor Cortex in Voluntary Movements: A Distributed System for Distributed Functions. Series: Methods and New Frontiers in Neuroscience. CRC Press, pp. 367401.

Pfurtscheller, G.; Leeb, R.; Keinrath, C.; Friedman, D.; Neuper, C.; Guger, C. \& Slater, M. (2006). Walking from thought, Brain Research, 1071, pp. 145-152.

Pfurtscheller, G. \& Neuper, C. (1997). Motor imagery activates primary sensorimotor area in man, Neurosci Lett, 239, pp. 65-68.

Ron-Angevin, R.; Estrella, A.D. \& Reyes-Lecuona, A. (2005). Development of a brainComputer Interface $(\mathrm{BCI})$ based on virtual reality to improve training technique, Applied Technologies in Medicine and Neuroscience, pp. 13-20.

Scherer, R.; Lee, F.; Schlögl, A.; Leeb, R.; Bischof, H. \& Pfurtscheller, G. (2007). Towards selfpaced (asynchronous) Brain-Computer Communication: Navigation through virtual worlds, IEEE Transactions on Biomedical Engineering, 2007.

Tech-on. (2006). To operate robot only with brain, ATR and Honda develop BMI base technology, 26 May 2006. 
Touyama, H. \& Hirose, M. (2007). Steady-state VEPs in CAVE for walking around the virtual world, Proc. of $12^{\text {th }}$ International Conference on Human-Computer Interaction, LNCS 4555, pp. 715-717.

Touyama, H. \& Hirose, M. (2007). Brain Computer Interface via Stereoscopic Images in CAVE, Proc. of $12^{\text {th }}$ International Conference on Human-Computer Interaction, LNCS 4557, pp. 1004-1007.

Trejo, L.J.; Rosipal, R. \&Matthews, B. (2006). Brain-computer interfaces for 1-D and 2-D cursor control: designs using volitional control of the EEG spectrum or steady-state visual evoked potentials, IEEE Trans. Neural. Syst. Rehabil. Eng, 14(2), Jun, pp. 225229.

Utsugi, K.; Obata, A.; Sato, H.; Katsura, T.; Sagara, K.; Maki, A. \& Koizumi, H. (2007). Development of an Optical Brain-machine Interface, Engineering in Medicine and Biology Society, 2007. EMBS 2007. 29th Annual International Conference of the IEEE Volume, Issue , 22-26 Aug. 2007 Page(s): 5338-5341.

Vapnik, V.N. (2000). The nature of statistical learning theory, Statistics for Engineering and Information Science, Springer-Verlag, New York.

Vidal, J.J. (1973). Towards direct brain-computer communication, Annu. Rev. Biophys. Bioeng, 2, pp. 157-180.

Watanabe, E., Yamashita, Y., Maki, A., Ito, Y. \& Koizumi, H. (1996). Non-invasive functional mapping with multi-channel near infra-red spectroscopic topography in humans. Neurosci Lett 205: pp. 41-44.

Wolpaw, J.R.; Birbaumer, N.; McFarland, D.J.; Pfurtscheller, G. \& Vaughan, T.M. (2002). Brain-computer interfaces for communication and control, Clinical Neurophysiology, 113, pp. 767-791.

Wolpaw, J.R. \& McFarland, D.J. (2004). Control of a two-dimensional movement signal by a noninvasive brain-computer interface in humans, PNAS, 101(51), pp. 17849-17854. 


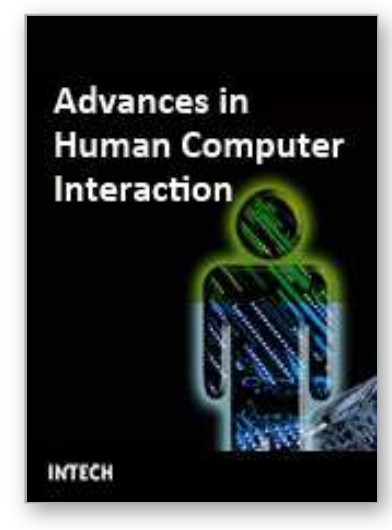

\section{Advances in Human Computer Interaction \\ Edited by Shane Pinder}

ISBN 978-953-7619-15-2

Hard cover, 600 pages

Publisher InTech

Published online 01, October, 2008

Published in print edition October, 2008

In these 34 chapters, we survey the broad disciplines that loosely inhabit the study and practice of humancomputer interaction. Our authors are passionate advocates of innovative applications, novel approaches, and modern advances in this exciting and developing field. It is our wish that the reader consider not only what our authors have written and the experimentation they have described, but also the examples they have set.

\section{How to reference}

In order to correctly reference this scholarly work, feel free to copy and paste the following:

Hideaki Touyama (2008). Brain-CAVE Interface Based on Steady-State Visual Evoked Potential, Advances in Human Computer Interaction, Shane Pinder (Ed.), ISBN: 978-953-7619-15-2, InTech, Available from: http://www.intechopen.com/books/advances_in_human_computer_interaction/braincave_interface_based_on_steady-state_visual_evoked_potential

\section{INTECH}

open science | open minds

\section{InTech Europe}

University Campus STeP Ri

Slavka Krautzeka 83/A

51000 Rijeka, Croatia

Phone: +385 (51) 770447

Fax: +385 (51) 686166

www.intechopen.com

\section{InTech China}

Unit 405, Office Block, Hotel Equatorial Shanghai

No.65, Yan An Road (West), Shanghai, 200040, China

中国上海市延安西路65号上海国际贵都大饭店办公楼 405 单元

Phone: +86-21-62489820

Fax: +86-21-62489821 
(C) 2008 The Author(s). Licensee IntechOpen. This chapter is distributed under the terms of the Creative Commons Attribution-NonCommercialShareAlike-3.0 License, which permits use, distribution and reproduction for non-commercial purposes, provided the original is properly cited and derivative works building on this content are distributed under the same license. 